\title{
Collective excitations in ferrimagnetic Heisenberg ladders
}

\author{
N. B. Ivanovt and J. Richter \\ Institut für Theoretische Physik, Universität Magdeburg, \\ P.O. Box 4120, D-39016 Magdeburg, Germany
}

(October 25, 2018)

\begin{abstract}
We study ground-state properties and the low-lying excitations of Heisenberg spin ladders composed of two ferrimagnetic chains with alternating site spins $\left(S_{1}>S_{2}\right)$ by using the bosonic DysonMaleev formalism and Lanczos numerical techniques. The emphasis is on properties of the ferrimagnetic phase which is stable for antiferromagnetic interchain couplings $J_{\perp} \geq 0$. There are two basic implications of the underlying lattice structure: (i) the spin-wave excitations form folded acoustic and optical branches in the extended Brillouin zone and (ii) the ground state parameters (such as the on-site magnetizations and spin-stiffness constant) show a crossover behavior in the weak-coupling region $0<J_{\perp}<1$. The above peculiarities of the ladder ferrimagnetic state are studied up to second order in the quasiparticle interaction and by a numerical diagonalization of ladders containing up to $N=12$ rungs. The presented results for the ground-state parameters and the excitation spectrum can be used in studies on the low-temperature thermodynamics of ferrimagnetic ladders.
\end{abstract}

PACS: 75.10.-b, 75.10.Jm, 75.40.Gb, 75.50.Gg

\section{INTRODUCTION}

There is an impressive difference between the properties of a single spin- $\frac{1}{2}$ antiferromagnetic Heisenberg chain and the related ladder system composed of two coupled chains. The spin- $\frac{1}{2}$ chain is critical, with a power-law decay of the spin correlations, whereas the spin- $\frac{1}{2}$ ladder has a finite energy gap in the excitation spectrum and exponentially decaying correlations. Similar behavior has been found in multi-leg spin- $\frac{1}{2}$ ladders with odd and even number of chains, respectively 2 Since in spin ladders the excitation spectrum is controlled by two energy scales (the intrachain exchange constant $J$ and the transverse interchain exchange coupling $J_{\perp}$ ), variation of $J_{\perp}$ and the applied magnetic field may produce a rich variety of specific quantum effects and phase transitions. In particular, intermediate plateaus in the magnetization processes of a number of ladder systems have been predifted and experimentally observed in the last few years.

Synthesized quasi-one-dimensional mixed-spin compounds 5 constitute an appropriate base for future developments in the physics of spin ladders. Most of them are molecular magnets containing two different transition-metal magnetic ions alternatively distributed on the lattice. Published experimental work implies that the magnetic properties of these mixed-spin materials are basically described by quantum Heisenberg spin models with antiferromagnetically coupled nearest-neighbor localized spins. Some typical examples of bipartite ladder structures composed of two different spins are shown in Fig. 1. The first two ladder structures reproduce, e.g., arrangements of the magnetic atoms $\operatorname{Mn}\left(S_{1}=\frac{5}{2}\right)$ and $\mathrm{Cu}\left(S_{2}=\frac{1}{2}\right)$ along the $a$-axis in the compounds $\mathrm{MnCu}(\mathrm{pbaOH})\left(\mathrm{H}_{2} \mathrm{O}\right)_{3}(\mathrm{pbaOH}=2$-hydroxy-1,3- propylenebisoxamato) and $\mathrm{MnCu}(\mathrm{pba})\left(\mathrm{H}_{2} \mathrm{O}\right)_{3} \cdot 2 \mathrm{H}_{2} \mathrm{O}$ (pba = 1,3-propylenebisoxamato), respectively. Along the $c$ - axis the magnetic ions in both mixed-spin compounds are arranged as shown in Fig. 11(c) 1 Since the molecular chemistry admits a relatively easy control of the molecular-unit positions, it may be expected that the discussed mixed-spin ladders will be synthesized in the near future.

a)

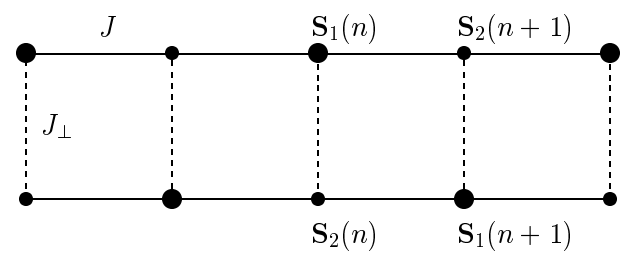

b)

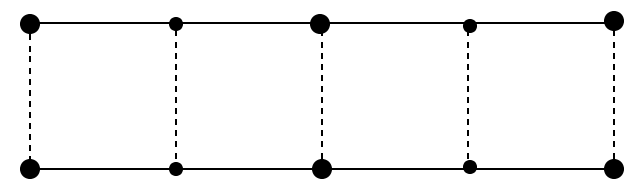

c)

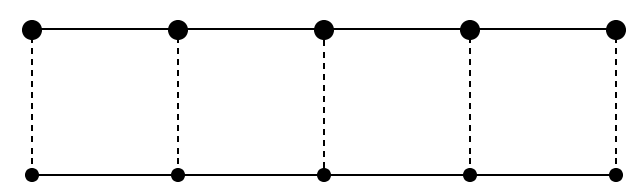

FIG. 1. Typical bipartite mixed-spin ladders composed of two types of site spins $\mathbf{S}_{1}(n)$ and $\mathbf{S}_{2}(n): \mathbf{S}_{1}^{2}(n)=S_{1}\left(S_{1}+1\right)$, $\mathbf{S}_{2}^{2}(n)=S_{2}\left(S_{2}+1\right)\left(S_{1}>S_{2}\right)$.

Ground-state properties of the Heisenberg spin models defined on the ladders shown in Fig. 1 have recently been studief by using semiclassical nonlinear model techniques found that an interplay between bond and spin alternations can produce various gapful phases separated by critical lines on the phase diagram. In this article we 
address the mixed-spin ladder model which is shown in Fig. 1(a) and defined by the Heisenberg Hamiltonian

$$
\begin{aligned}
\mathcal{H} & =J \sum_{n=1}^{N} \mathbf{S}_{1}(n) \cdot \mathbf{S}_{2}(n+1)+\mathbf{S}_{2}(n) \cdot \mathbf{S}_{1}(n+1) \\
& +J_{\perp} \sum_{n=1}^{N} \mathbf{S}_{1}(n) \cdot \mathbf{S}_{2}(n)
\end{aligned}
$$

The integers $n$ label $N$ rungs, each of them containing two different site spins with quantum spin numbers $S_{1}>S_{2}$. Below we frequently use the notations $\sigma=S_{1} / S_{2}>1$ and $S_{2}=S$, and set $J=1$.

For antiferromagnetic interchain couplings $\left(J_{\perp}>0\right)$, the model (11) exhibits a ferrimagnetic ground state characterized by the net ferromagnetic moment $\left(S_{1}-S_{2}\right) N .10$ Such a magnetic phase may be referred to as a quantized unsaturated ferromagnetic phase: it is characterized both by the quantized ferromagnetic order parameter $\mathbf{M}=\sum_{n=1}^{N}\left[\mathbf{S}_{1}(n)+\mathbf{S}_{2}(n)\right]$ [quantized in integral or half-integral multiples of the number of rungs, $M=$ $\left.\left(S_{1}-S_{2}\right) N\right]$ and by the macroscopic sublattice magnetizations $M_{A}=\sum_{n=1}^{N}\left\langle S_{1}^{z}(n)\right\rangle$ and $M_{B}=\sum_{n=1}^{N}\left\langle S_{2}^{z}(n)\right\rangle$. In the limit $J_{\perp} \rightarrow \infty$ the rung spins form local spin states with a total spin $S_{t o t}=S_{1}-S_{2}$ : in particular, the $\left(1, \frac{1}{2}\right)$ ladder is equivalent to the spin- $\frac{1}{2}$ ferromagnetic Heisenberg model with an effective exchange interaction $J_{\text {eff }}=-\frac{8}{9}$. On the other hand, for ferromagnetic interchain couplings $\left(J_{\perp}<0\right)$, it may be generally expected a magnetically disordered spin-singlet ground state, since the isotropic Heisenberg model (11) is defined on a bipartite one-dimensional (1D) lattice.

In spite of the fact that the ferrimagnetic long-range order exists already in the limit of noninteracting chains, in the antiferromagnetic region $\left(J_{\perp}>0\right)$ the checkerboard ladder exhibits various specific properties deserving a special study. As shown below, the most interesting region corresponds to relatively small interchain couplings $\left(0<J_{\perp}<1\right)$ where a number of ground-state parameters exhibit a crossover behavior: the latter is marked by the extreme values of these parameters. For instance, in that region the sublattice magnetization $M_{A}$ and the spinstiffness constant $\rho_{s}$ reach their maximal values. Another specific property of the ferrimagnetic ladder concerns the collective spin-wave excitations, which form in the extended Brillouin zone $-\pi / a_{0}<k<\pi / a_{0}$ folded acoustic and optical branches characterized by a minimum at the zone boundary $k=\pi / a_{0}$ and a maximum between $k=\pi /\left(2 a_{0}\right)$ (for $J_{\perp}=0$ ) and $k=\pi / a_{0}$ (for $\left.J_{\perp}=\infty\right), a_{0}$ being the lattice spacing along the ladder. A purpose of the present work is to analyze the above mentioned peculiarities of the ladder system.

The existence of a magnetic ground state opens the possibility of using systematic spin-wave approaches for the 1D Hamiltopian (1). Indeed, recently published calculations 1113 argued that the spin-wave theory (SWT) is capable to produce precise quantitative results both for the ground-state parameters and excitation spectrum of ferrimagnetic Heisenberg chains, including the extreme quantum system $\left(1, \frac{1}{2}\right)$. Turning to the ferrimagnetic ladders, a SWT description in the weak-coupling limit $J_{\perp} \ll 1$ looks to some extend puzzling since in that region one may expect strong fluctuations of the macroscopic chain magnetizations around the common quantization axis. Similar problems arise when the semiclassical nonlinear $\sigma$ model technique is applied to antiferromagnetic spin ladders.14 A successful treatment of the weak-coupling limit $J_{\perp} \ll 1$ in ferrimagnetic ladders requires an appropriate choice of the free-quasiparticle bosonic Hamiltonian. Using as a guide the Lanczos numerical technique, it is argued below that the Dyson-Maleev formalism is capable to produce precise quantitative description of the ferrimagnetic state in a relatively large range of interchain couplings, including the weak-coupling limit $J_{\perp} \ll 1$.

The paper is organized as follows. In Section 2 we introduce the bosonic representation of $\mathcal{H}$ and discuss the choice of an appropriate free-quasiparticle bosonic Hamiltonian. As a criterion we use the perturbation series for $M_{A}$ compared to Lanczos numerical results. In Section 3 we study the excitation spectrum of the model up to second order in the bosonic interaction $V$. Section 4 contains a summary of the results and discussions concerning also the disordered phase. In the Appendix we calculate the second-order $\mathcal{O}\left(V^{2}\right)$ corrections for $M_{A}$.

\section{BOSONIC REPRESENTATION OF $\mathcal{H}$ : PERTURBATION SERIES FOR $M_{A}$}

\section{A. Bosonic Hamiltonian}

We adopt the Dyson-Maleev formalism.15 Performing subsequently the Dyson-Maleev, Fourier, and Bogoliubov transformations, we obtain the following Hamiltonian in terms of the quasiparticle bosonic operators $\alpha_{k}$ and $\beta_{k}: 16$

$$
\mathcal{H}_{B}=E_{0}+\mathcal{H}_{0}+V, \quad V=V_{2}+V_{D M}
$$

$E_{0}$ is the ground-state energy of the ferrimagnetic state calculated up to first order in $1 / S$ (see Fig. 2):

$$
\begin{aligned}
\frac{E_{0}}{N} & =-\left(1+\frac{J_{\perp}}{2}\right)\left[2 \sigma S^{2}+S(1+\sigma)\left(1-\frac{1}{N} \sum_{k} \varepsilon_{k}\right)\right] \\
& -2\left(c_{1}^{2}+c_{2}^{2}\right)-J_{\perp}\left(c_{1}^{2}+c_{3}^{2}\right) \\
& -\left(2 c_{2}+J_{\perp} c_{3}\right) c_{1} \frac{\sigma+1}{\sqrt{\sigma}},
\end{aligned}
$$

where

$$
\begin{aligned}
& c_{1}=-\frac{1}{2}+\frac{1}{2 N} \sum_{k} \frac{1}{\varepsilon_{k}}, \quad c_{2}=-\frac{1}{2 N} \sum_{k} \gamma_{k} \frac{\eta_{k}}{\varepsilon_{k}} \\
& c_{3}=-\frac{1}{2 N} \sum_{k} \frac{\eta_{k}}{\varepsilon_{k}}, \quad \varepsilon_{k}=\sqrt{1-\eta_{k}^{2}}
\end{aligned}
$$




$$
\eta_{k}=\frac{2 \sqrt{\sigma}}{\sigma+1} \frac{J_{\perp} / 2+\gamma_{k}}{J_{\perp} / 2+1}, \quad \gamma_{k}=\cos \left(k a_{0}\right)
$$

The sums run over the wave vectors $k$ from the lattice Brillouin zone $-\pi / a_{0} \leq k \leq \pi / a_{0}$.

$\mathcal{H}_{0}$ is the usual quadratic quasiparticle Hamiltonian of the linear spin-wave theory (LSWT) corrected by the diagonal quadratic terms coming from a normal ordering of the quartic Dyson-Maleev bosonic interaction. It is important that these corrections (similar to Oguchi's corrections in antiferromagnets 17 ) renormalize the magnon excitation spectrum (and the ground-state energy) without changing its basic structure, i.e., the number of Goldstone modes. In terms of quasiparticle operators the Hamiltonian $\mathcal{H}_{0}$ simply reads

$$
\mathcal{H}_{0}=2 S \sum_{k} \omega_{k}^{(\alpha)} \alpha_{k}^{\dagger} \alpha_{k}+\omega_{k}^{(\beta)} \beta_{k}^{\dagger} \beta_{k}
$$

where

$$
\begin{aligned}
\omega_{k}^{(\alpha, \beta)} & =\left(1+\frac{J_{\perp}}{2}\right)\left(\frac{\sigma+1}{2} \varepsilon_{k} \mp \frac{\sigma-1}{2}\right) \\
& +\frac{C_{k} \eta_{k}-D}{4 S \varepsilon_{k}} \pm \frac{\sigma-1}{4 S \sqrt{\sigma}}\left(2 c_{2}+c_{3} J_{\perp}\right)
\end{aligned}
$$

$C_{k}=c_{1}(\sigma+1)\left(2 \gamma_{k}+J_{\perp}\right) / \sqrt{\sigma}+2\left(2 c_{2} \gamma_{k}+c_{3} J_{\perp}\right)$, and $D=2 c_{1}\left(2+J_{\perp}\right)+(\sigma+1)\left(2 c_{2}+J_{\perp} c_{3}\right) / \sqrt{\sigma}$. Here $\omega_{k}^{(\alpha, \beta)}$ are the dressed quasiparticle dispersions. The functions $\omega_{k}^{(\alpha, \beta)}$ without $\mathcal{O}(1 / S)$ corrections will be referred to as bare dispersions.

Finally, the quasiparticle interaction $V$ includes two different terms: the quadratic bosonic interaction

$$
V_{2}=\sum_{k} V_{k}^{(+)} \alpha_{k}^{\dagger} \beta_{k}^{\dagger}+V_{k}^{(-)} \alpha_{k} \beta_{k}
$$

which is defined by the vertex functions

$$
V_{k}^{( \pm)}=\frac{D \eta_{k}-C_{k}}{2 \varepsilon_{k}} \mp \frac{\sigma-1}{\sqrt{\sigma}} c_{1}\left(\gamma_{k}+\frac{J_{\perp}}{2}\right)
$$

and the quartic Dyson-Maleev interaction $V_{D M}$ defined by the vertex functions $V^{(i)}=V_{12 ; 34}^{(i)}, i=1, \ldots, 9.18$ Here and in what follows we use the wave-vector abbreviations $\left(k_{1}, k_{2}, k_{3}, k_{4}\right) \equiv(1,2,3,4)$.

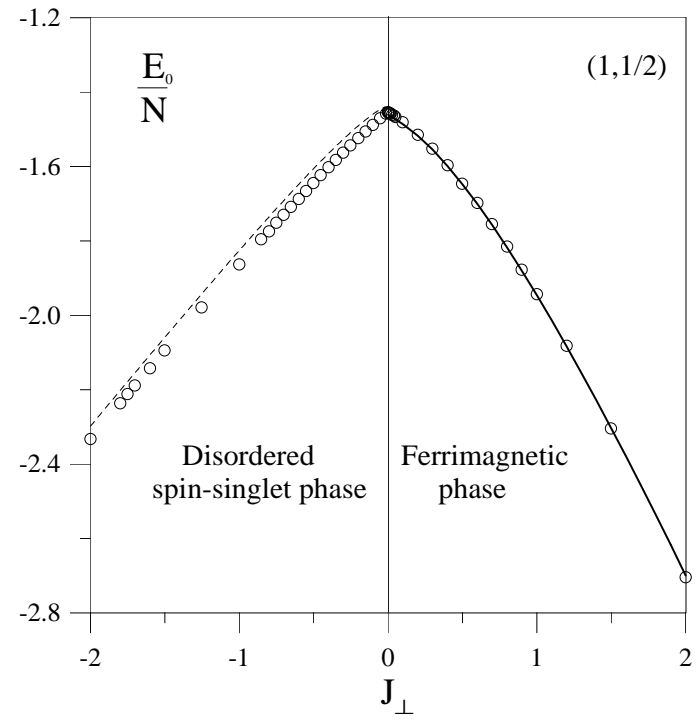

FIG. 2. Ground-state energy of the $\left(1, \frac{1}{2}\right)$ ladder as a function of the interchain coupling $J_{\perp}$. The solid line displays the first-order $\mathcal{O}(1 / S)$ spin-wave result for the ferrimagnetic state, Eq. (3). The dashed line indicates the ground-state energy of the disordered spin-singlet phase, as obtained from the LSWT. The Lanczos numerical results for a ladder with $N=10$ rungs are denoted by open circles. At $J_{\perp}=0$ there is a well pronounced cusp in the data indicating a sharp transition from the ferrimagnetic state into a disordered spin-singlet state.

\section{B. Perturbation series for $M_{A}$}

In spite of the fact that the expression for the groundstate energy (3) produces an excellent fit to the exactdiagonalization (ED) results in a large interval up to $J_{\perp}=10$ (in Fig. 2 we show a smaller interval), this fact by itself is not enough to make a statement about the accuracy of the SWT. It is more appropriate to use as a criterion the sublattice magnetization $M_{A}$, since the latter keeps information on the long-range spin correlations as well. The zeroth-order $\mathcal{O}\left(V^{0}\right)$ result for $m_{A}=M_{A} / N$ simply reads $m_{A}=S_{1}-c_{1}$. We shall deal below only with the on-site magnetization $m_{A}$, as the relation $m_{A}+m_{B}=S_{1}-S_{2}\left(m_{B}=M_{B} / N\right)$ is fulfilled up to an arbitrary order of the perturbation series. Note that in the antiferromagnetic case $S_{1}=S_{2}$ the offdiagonal interaction $V_{2}$ in $\mathcal{H}_{B}$ disappears and, as a result, there are no $\mathcal{O}(V)$ corrections to $m_{A}$. In the ferrimagnetic case $V_{2} \neq 0$, so that the expansion for $m_{A}$ in powers of $V$ has the form

$$
m_{A}=S_{1}-c_{1}-\frac{1}{4 S N} \sum_{k} \frac{\eta_{k}}{\varepsilon_{k}} \frac{V_{k}^{(+)}+V_{k}^{(-)}}{\omega_{k}^{(\alpha)}+\omega_{k}^{(\beta)}}+\mathcal{O}\left(V^{2}\right) .
$$

In Fig. 3 we show results for $m_{A}$ obtained from Eq. (8) by using subsequently the bare and dressed dispersions 
$\omega_{k}^{(\alpha, \beta)}$ from Eq. (5). Comparing with the numerical results, one indicates that the expansion in powers of $1 / S$ (using bare dispersions) does not describe qualitatively the weak-coupling limit $J_{\perp} \ll 1$ : it predicts a small decrease of $m_{A}$ in the vicinity of $J_{\perp}=0$. On the other hand, we find that the expansion in powers of $V$ (using dressed dispersions) gives a correct qualitative result in the weak-coupling limit $J_{\perp} \ll 1$ as well. The indicated problem of the standard $1 / S$ expansion might be a result of the enhanced fluctuations of the chain magnetizations about the common quantization axis, and/or of the usual zero-point fluctuations [as far as the extreme quantum system $\left(1, \frac{1}{2}\right)$ is concerned]. The above observation implies that at least in the extreme quantum case $\left(1, \frac{1}{2}\right)$ the expansion in powers of $V$ is more reliable: such a viewpoint is strongly supported by the second-order $\mathcal{O}\left(V^{2}\right)$ result for $m_{A}$ (see the Appendix and Fig. 3). The above interpretation of the spin-wave series (as power series in $V$ ) is also adopted in the following analysis of the excitation spectrum. As a matter of fact, the difference between the standard $1 / S$ expansion and those in powers of $V$ is mostly pronounced in the extreme quantum case $\left(1, \frac{1}{2}\right)$ : for larger site spins both expansions practically coincide.

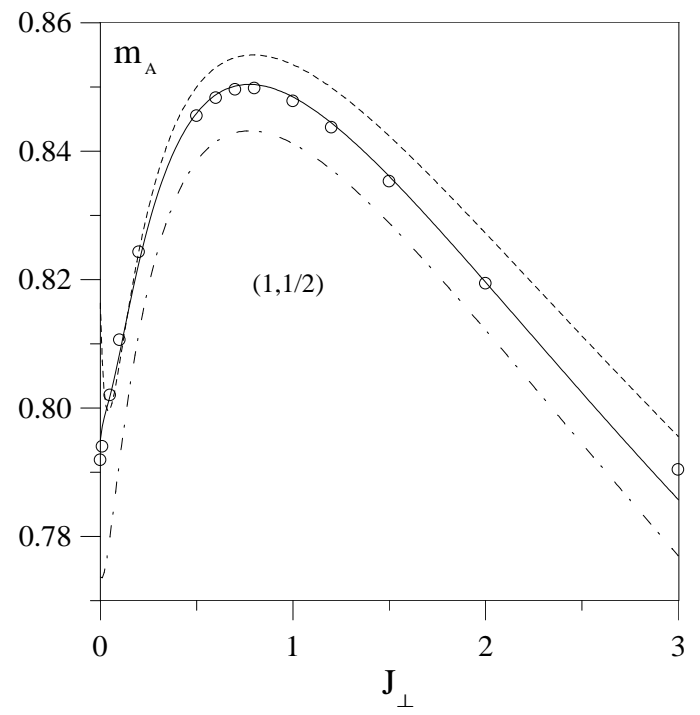

FIG. 3. On-site magnetization (sublattice $\mathcal{A}$ ) of the ferrimagnetic phase as a function of the interchain coupling $J_{\perp}$. The dashed and dashed-dotted lines display the series results up to first order in powers of $1 / S$ (bare dispersions) and $V$ (dressed dispersions), respectively [Eq. (8)]. The solid line shows the second-order $\mathcal{O}\left(V^{2}\right)$ series result for $m_{A}$ (see the Appendix). The Lanczos numerical results for the $N=12$ ladder are denoted by open circles.

In the whole interval $0 \leq J_{\perp} \leq 3$ shown in Fig. 3 , the second-order result for $m_{A}$ differs by less than $0.3 \%$ from the ED estimates based on the $N=12$ ladder 19 . For larger $J_{\perp}$ one clearly indicates a monotonic increase of the deviations from the numerical estimates, which can be ascribed to the enlarged reduction of the classical spin $\delta S_{1}=S_{1}-m_{A}$ : in the limit $J_{\perp} \rightarrow \infty$ the exact result is $\delta S_{1}=\frac{1}{3}$. It is shown in the next Section that the SWT description of the excitation spectrum exhibits similar precision but in a smaller parameter range $\left(0 \leq J_{\perp} \leq 2\right)$

\section{EXCITATION SPECTRUM}

\section{A. Free quasiparticles}

The quadratic Hamiltonian $\mathcal{H}_{0}$ defines two branches of spin-wave excitations ( $\alpha$ and $\beta$ magnons) described by the dispersion relations $E_{k}^{(\alpha, \beta)}=2 S \omega_{k}^{(\alpha, \beta)}$, Eq. (5), in the extended Brillouin zone $-\pi / a_{0} \leq k \leq \pi / a_{0}$ (see Fig. (4). The excited states composed of $\alpha$ and $\beta$ magnons belong to subspaces with $M^{z} \leq\left(S_{1}-S_{2}\right) N-1$ and $M^{z} \geq\left(S_{1}-S_{2}\right) N+1$, respectively. The magnon energies $E_{k}^{(\alpha, \beta)}$ are calculated up to the order $\mathcal{O}(1 / S)$. In the long wavelength limit $k a_{0} \ll 1$, the ferromagnetic branch $E_{k}^{(\alpha)}$ has the quadratic Landau-Lifshits form

$$
E_{k}^{(\alpha)}=\frac{\rho_{s}}{M_{0}} k^{2}+\mathcal{O}\left(k^{4}\right)
$$

where $M_{0}=\left(S_{1}-S_{2}\right) /\left(2 a_{0}\right)$ and $\rho_{s}$ are, respectively, the magnetization density per chain and the spin-stiffness constant 20 of the ferrimagnetic ladder. This form of the Goldstone modes is characteristic for Heisenberg ferromagnets and reflects the fact that the order parameter (i.e., the ferromagnetic moment) is itself a constant of the motion.20 An alternative approach, relying on the expected conformal invariance of the related XXZ ladder model, may also be used to predict the quadratic form of Eq. (9).21

The spin-stiffness constant $\rho_{s}$ and the magnetization density $M_{0}$ play a basic role in the low-temperature thermodynamics of 1D models with a continuous symmetry and conserved order parameters.22 Up to the order $\mathcal{O}(1 / S), \rho_{s}$ can be obtained from the Landau-Lifshitz relation (9) and Eq. (5):

$$
\frac{\rho_{s}}{a_{0} S_{1} S_{2}}=1-c_{1} \frac{\sigma+1}{S \sigma}-\frac{c_{2}}{S \sqrt{\sigma}} .
$$

At the zone boundary $k=\pi / a_{0}$ the ferromagnetic branch exhibits an additional minimum so that in the vicinity of $\pi / a_{0}$ the spectrum reads

$$
E_{k}^{(\alpha)}=\Delta_{\pi}^{(\alpha)}+\operatorname{const}\left(\frac{\pi}{a_{0}}-k\right)^{2}
$$

where $\Delta_{\pi}^{(\alpha)}$ is the excitation gap at the zone boundary. Such a branch folding of the excitation spectrum is typical for ladder structures - it_has been indicated in uniform spin- $\frac{1}{2}$ ladders as well.23 The excitation mode at $k=\pi / a_{0}$ reflects the underlying ladder structure and in the weak-coupling limit $J_{\perp} \ll 1$ may be interpreted 
as uniform rotations of the antiferromagnetic moment $\mathbf{L}=\sum_{n=1}^{N}(-1)^{n+1}\left[\mathbf{S}_{1}(n)-\mathbf{S}_{2}(n)\right]$. As $J_{\perp} \rightarrow 0$, the excitation gap $\Delta_{\pi}^{(\alpha)} \propto J_{\perp}$ goes to zero. For ferromagnetic couplings $J_{\perp}<0$, the $k=\pi / a_{0}$ mode becomes unstable, thus producing a global instability of the ferrimagnetic state. The minimum at $k=\pi / a_{0}$ persists up to the limit $J_{\perp}=\infty$ : the related maximum of $E_{k}^{(\alpha)}$ changes its position from $k=\pi /\left(2 a_{0}\right)$ (for $J_{\perp}=0$ ) to $k=\pi / a_{0}$ (for $\left.J_{\perp}=\infty\right)$.

Similar folding appears in the optical antiferromagnetic branch $E_{k}^{(\beta)}$, which can be characterized by the spectral gaps $\Delta_{0}^{(\beta)}$ and $\Delta_{\pi}^{(\beta)}$ at $k=0$ and $k=\pi$, respectively. For instance, using Eq. (5), the spectral gap $\Delta_{0}^{(\beta)}$ up to $\mathcal{O}(1 / S)$ reads

$$
\Delta_{0}^{(\beta)}=\left(2+J_{\perp}\right)\left(S_{1}-S_{2}\right)\left(1-\frac{2 c_{2}+c 3 J_{\perp}}{S \sqrt{\sigma}\left(2+J_{\perp}\right)}\right) .
$$

For the $\left(1, \frac{1}{2}\right)$ ladder at $J_{\perp}=0$, Eqs. (10) and (12) give the estimates $\rho_{s} /\left(a_{0} S_{1} S_{2}\right)=0.761$ and $\Delta_{0}^{(\beta)}=1.676$. On the other hand, the LSWT Hamiltonian $\mathcal{H}_{0}^{\prime}$ produces the parameters of the related classical system: $\rho_{s} /\left(a_{0} S_{1} S_{2}\right)=1$ and $\Delta_{0}^{(\beta)}=1$. The numerical estimate for the gap $\Delta_{0}^{(\beta)}=1.759$ at $J_{\perp}=024$ clearly demonstrates the importance of the $1 / S$ corrections in Eq. (5).

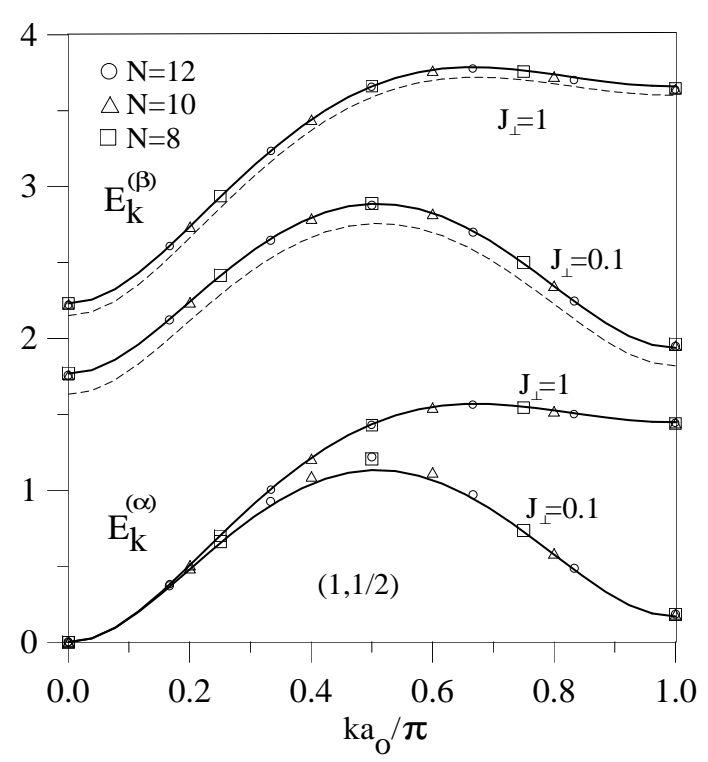

FIG. 4. Excitation spectrum of the $\left(1, \frac{1}{2}\right)$ ladder for interchain couplings $J_{\perp}=0.1$ and $J_{\perp}=1$. The dashed and solid lines display, respectively, the series results up to the orders $\mathcal{O}\left(V^{0}\right) \equiv \mathcal{O}(1 / S)$ and $\mathcal{O}\left(V^{2}\right)$. The $\mathcal{O}\left(V^{0}\right)$ results for the acoustic branch $E_{k}^{(\alpha)}$ (which are not displayed) closely follow the presented second-order curves. The symbols indicate our Lanczos numerical results.

\section{B. Quasiparticle interactions: second-order corrections for $\omega_{k}^{(\alpha, \beta)}$}

The quasiparticle interaction $V$ produces corrections to the dispersions $\omega_{k}^{(\alpha, \beta)}$ only up from the order $\mathcal{O}\left(V^{2}\right)$. The second-order corrections for the ferromagnetic branch $\omega_{k}^{(\alpha)}$ are described by the self-energy diagrams shown in Fig. 2 of Ref. 13. The respective analytic expression for the corrections reads

$$
\begin{aligned}
\delta \omega_{k}^{(\alpha)} & =-\frac{1}{(2 S)^{2}}\left[\frac{V_{k}^{(+)} V_{k}^{(-)}}{\omega_{k}^{(\alpha)}+\omega_{k}^{(\beta)}}\right. \\
& -\frac{2}{N} \sum_{p} \frac{V_{p}^{(+)} V_{k p ; p k}^{(2)}+V_{p}^{(-)} V_{k p ; p k}^{(3)}}{\omega_{p}^{(\alpha)}+\omega_{p}^{(\beta)}} \\
+ & \frac{2}{N^{2}} \sum_{2-4} \delta_{k 2}^{34} \frac{V_{43 ; 2 k}^{(8)} V_{k 2 ; 34}^{(7)}}{\omega_{k}^{(\alpha)}+\omega_{2}^{(\alpha)}+\omega_{3}^{(\beta)}+\omega_{4}^{(\beta)}} \\
+ & \left.\frac{2}{N^{2}} \sum_{2-4} \delta_{k 2}^{34} \frac{V_{43 ; 2 k}^{(3)} V_{k 2 ; 34}^{(2)}}{-\omega_{k}^{(\alpha)}+\omega_{2}^{(\beta)}+\omega_{3}^{(\alpha)}+\omega_{4}^{(\alpha)}}\right] .
\end{aligned}
$$

Note that since the vertex functions $V_{k}^{(-)}, V_{k p ; p k}^{(2)}, V_{k p ; p k}^{(3)}$, $V_{43 ; 2 k}^{(8)}$, and $V_{43 ; 2 k}^{(3)}$ vanish at the zone center $k=0$, the gapless structure of $\omega_{k}^{(\alpha)}$ is preserved separately by each diagram $25 \delta_{12}^{34} \equiv \delta(1+2-3-4)$ is the Kronecker $\delta$ function.

The second-order corrections for $\omega_{k}^{(\beta)}$ come from similar diagrams. The explicit expression reads

$$
\begin{aligned}
& \delta \omega_{k}^{(\beta)}=-\frac{1}{(2 S)^{2}}\left[\frac{V_{k}^{(+)} V_{k}^{(-)}}{\omega_{k}^{(\alpha)}+\omega_{k}^{(\beta)}}\right. \\
& -\frac{2}{N} \sum_{p} \frac{V_{p}^{(+)} V_{k p ; p k}^{(5)}+V_{p}^{(-)} V_{k p ; p k}^{(6)}}{\omega_{p}^{(\alpha)}+\omega_{p}^{(\beta)}} \\
& +\frac{2}{N^{2}} \sum_{2-4} \delta_{k 2}^{34} \frac{V_{43 ; 2 k}^{(7)} V_{k 2 ; 34}^{(8)}}{\omega_{k}^{(\beta)}+\omega_{2}^{(\beta)}+\omega_{3}^{(\alpha)}+\omega_{4}^{(\alpha)}} \\
& \left.+\frac{2}{N^{2}} \sum_{2-4} \delta_{k 2}^{34} \frac{V_{43 ; 2 k}^{(5)} V_{k 2 ; 34}^{(6)}}{-\omega_{k}^{(\beta)}+\omega_{2}^{(\alpha)}+\omega_{3}^{(\beta)}+\omega_{4}^{(\beta)}}\right] .
\end{aligned}
$$

The above expressions can be used to find the secondorder corrections for the spin-stiffness constant $\rho_{s}$ and the excitation gaps $\Delta_{\pi}^{(\alpha)}, \Delta_{0}^{(\beta)}$, and $\Delta_{\pi}^{(\beta)}$. The results for the ferrimagnetic state are summarized in Figs. 4 -6 and in Table II 


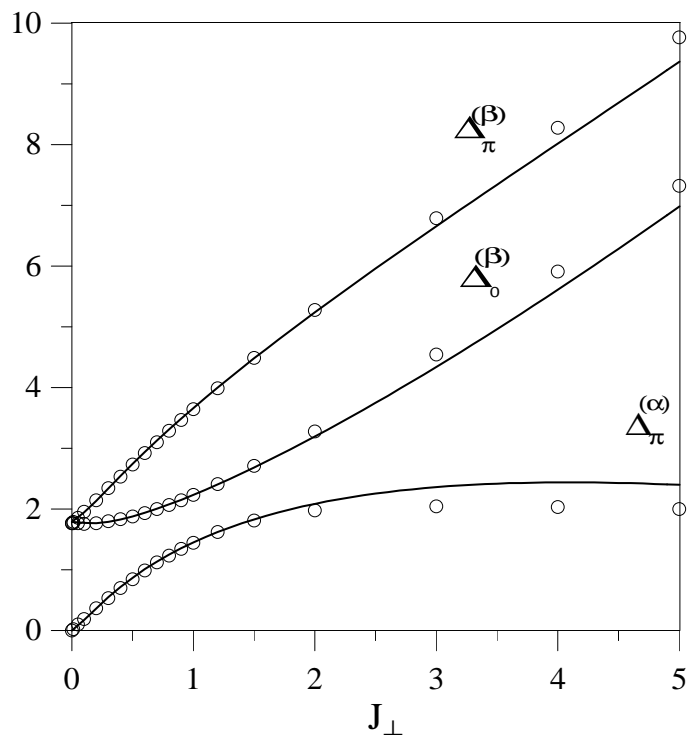

FIG. 5. Gaps in the excitation spectrum of the $\left(1, \frac{1}{2}\right)$ ferrimagnetic ladder as functions of the interchain coupling $J_{\perp}$. The solid lines represent perturbation series results up to second order in $V$. The open circles indicate Lanczos numerical results for a ladder with $N=12$ rungs.

\section{ANALYSIS OF THE RESULTS AND CONCLUSIONS}

A comparison of the SWT and ED results (see Fig. 国) implies that already at the level of free quasiparticles the theoretical curves for the acoustic branch $E_{k}^{(\alpha)}$ closely follow the numerical estimates almost in the whole Brillouin zone apart from some vicinity of the wave vector $k=\pi /\left(2 a_{0}\right)$. Note that the indicated discrepancy exists only in the weak-coupling limit (the case $J_{\perp}=0.1$ in Fig. (1). Similar problem has been indicated for the $\left(1, \frac{1}{2}\right)$ ferrimagnetic chain 13 The point is that the secondorder corrections for $E_{k}^{(\alpha)}$, Eq. (13), in the vicinity of $k=\pi /\left(2 a_{0}\right)$ are very small (about $0.6 \%$ from the principal approximation for $\left.J_{\perp}=0.1\right)$. Therefore, in the weak-coupling limit $J_{\perp} \ll 1$ the series does not describe in a proper way the acoustic branch close to the wave vector $k=\pi /\left(2 a_{0}\right)$ (where the deviation is about $10 \%$ from the extrapolated ED result). Looking at the recently published Monte Carlo results for different $\left(S_{1}, S_{2}\right)$ ferrimagnetic chain 12 , it is seen that the discussed discrepancy in the region of $k=\pi /\left(2 a_{0}\right)$ is well pronounced only in the extreme quantum system $\left(1, \frac{1}{2}\right)$. On the other hand, turning to the optical branch $E_{k}^{(\beta)}$, one finds an excellent agreement between the second-order $\mathcal{O}\left(V^{2}\right)$ perturbation result and the numerical estimates (see Fig. (4). For instance, the theoretical result for the excitation gap $\Delta_{0}^{(\beta)}$ at $J_{\perp}=0.1$ differs by less than $0.5 \%$ from the ED estimate. Moreover, it can be expected 13 that the thirdorder corrections further improve the above result. 26 The plots of the excitation gaps $\Delta_{\pi}^{(\alpha)}, \Delta_{0}^{(\beta)}$, and $\Delta_{\pi}^{(\beta)}$ (see Fig. 5) can be used to find the range where the SWT still produces good quantitative results. The largest discrepancies are connected with the excitation gap $\Delta_{\pi}^{(\alpha)}$ (at $J_{\perp}=1.5$ the deviation from the ED result is $1.4 \%$, but at $J_{\perp}=3$ it already exceeds $15 \%$ ). The discrepancies grow with the interchain interaction, but up to $J_{\perp} \approx 2$ the theory produces good quantitative results.

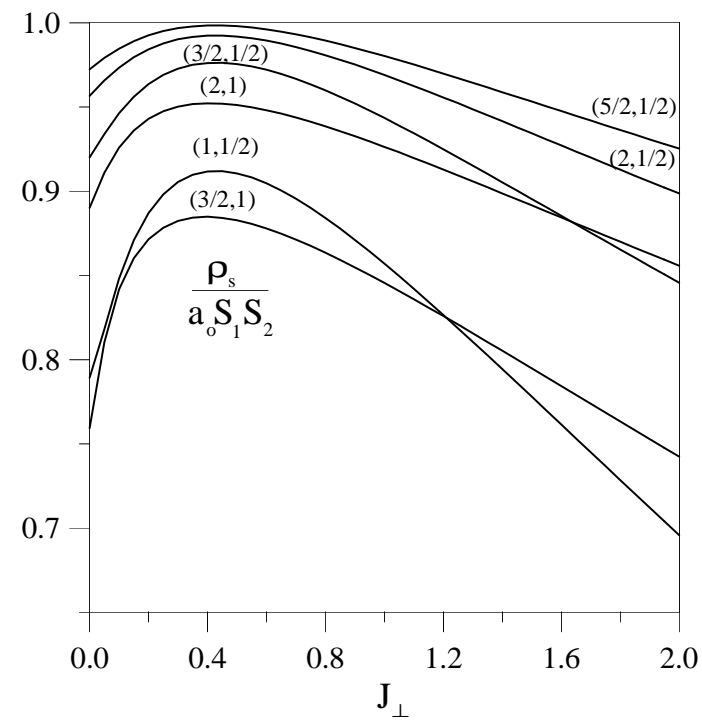

FIG. 6. Spin-stiffness constants versus $J_{\perp}$ of different $\left(S_{1}, S_{2}\right)$ ferrimagnetic ladders calculated up to second order of the perturbation series in $V$.

The plots of $m_{A}, \Delta_{0}^{(\beta)}$, and $\rho_{s}$ (see Figs. 3, 5, and 6) also show that the short-range correlations, which are typical for the strong-coupling limit $J_{\perp} \rightarrow+\infty$, are qualitatively established already at $J_{\perp}=2$. On the other hand, the same plots indicate a well pronounced crossover behavior for smaller interchain couplings $\left(0<J_{\perp}<1\right)$ : the spin-stiffness constant $\rho_{s}$ and the on-site magnetization $m_{A}$ reach their maximal values (respectively, at $J_{\perp}=0.45$ and 0.77$)$, whereas the excitation gap $\Delta_{0}^{(\beta)}$ becomes minimal (at $J_{\perp} \approx 0.15$ ). The discussed peculiarities appear as a result of the crossover between different types of short-range correlations without global change of the ground-state symmetry.

Turning to the case of ferromagnetic interchain couplings $\left(J_{\perp}<0\right)$, as mentioned above, one can expect that the model (11) is in a disordered spin-singlet phase. In the limit $J_{\perp} \rightarrow-\infty$, the system is equivalent to the antiferromagnetic spin- $\left(S_{1}+S_{2}\right)$ Heisenberg chain. Again the weak-coupling limit $\left|J_{\perp}\right| \ll 1$ is more interesting, especially in the presence of exchange anisotropies. Here we shall restrict ourselves to short comments on the weakcoupling limit $\left|J_{\perp}\right| \ll 1$ of the isotropic $\left(1, \frac{1}{2}\right)$ ladder. The ED results is Figs. I and 8 imply that already in the weak-coupling limit $\left|J_{\perp}\right| \ll 1$ the short-range spin correlations qualitatively reproduce the corresponding spin 
correlations in the spin- $\frac{3}{2}$ antiferromagnetic Heisenberg chain. A qualitative LSWT analysis of the excitation spectrum predicts two degenerate branches of spin-wave excitations $E_{k}^{( \pm)}$. The lower branch $E_{k}^{(-)}$has a linear dispersion close to the wave vector $k=0$, which is characterized by the spin-wave velocity

$$
c_{s w}=\frac{4 S_{1} S_{2} a_{0}\left|J_{\perp}\right|^{1 / 2}}{\sqrt{\left|J_{\perp}\right|\left(S_{1}+S_{2}\right)^{2}+2\left(S_{1}-S_{2}\right)^{2}}} .
$$

On the other hand, $E_{k}^{(+)}$describes gapped spin-wave excitations: the characteristic spectral gap at $k=0$ is

$$
\Delta=\sqrt{\left|J_{\perp}\right|^{2}\left(S_{1}+S_{2}\right)^{2}+\left|J_{\perp}\right|\left(S_{1}^{2}+S_{2}^{2}\right)+4\left(S_{1}-S_{2}\right)^{2}} .
$$

It is seen that the relativistic modes remain stable (and the gap $\Delta$ is finite) up to the limit of noninteracting chains. The above LSWT result may be interpreted in the sense that the classical magnetic state in the region
$J_{\perp}<0$ is swept out by the quantum fluctuations.27 On the other hand, as is well known, a LSWT analysis cannot exclude the existence of gapped Haldane-type phases. To some extend, the discussed ferrimagnetic model $\left(J_{\perp}<0\right)$ in the weak-coupling limit resembles the recently studied two-leg spin ladder constructed of ferromagnetic spin- $\frac{1}{2}$ chains with antiferromagnetic interchain couplings $\left(J_{\perp}>0\right) .28 .29$ It was found that for arbitrary $J_{\perp}>0$ and isotropic exchange interactions the ground state is disordered and the system behaves like the spin-1 Heisenberg antiferromagnetic chain. Although the above analysis cannot completely exclude the existence of a similar gapped phase for $\left|J_{\perp}\right| \ll 1$, we believe that the critical phase remains stable up to $J_{\perp}=0^{-}$. Such a conclusion is supported by the ED results which demonstrate well-established short-range correlations even in the limit $\left|J_{\perp}\right| \ll 1$. In terms of the related nonlinear $\sigma$ model description, on may expect that one and the same phase $\theta=\pi(\bmod 2 \pi)$ of the topological term will describe the whole parameter range $J_{\perp}<0$.

TABLE I. Results for the spin-stiffness constant $\rho_{s}$, the on-site magnetization $m_{A}$, and the excitation gaps $\left(\Delta_{\pi}^{(\alpha)}, \Delta_{0}^{(\beta)}\right.$, and $\Delta_{\pi}^{(\beta)}$ ) of different $J_{\perp}=1$ ferrimagnetic ladders, as obtained from the second-order perturbation series in $V$.

\begin{tabular}{lccccc}
\hline \hline$\left(S_{1}, S_{2}\right)$ & $\rho_{s} /\left(a_{0} S_{1} S_{2}\right)$ & $m_{A}$ & $\Delta_{\pi}^{(\alpha)}$ & $\Delta_{0}^{(\beta)}$ & $\Delta_{\pi}^{(\beta)}$ \\
\hline$\left(1, \frac{1}{2}\right)$ & 0.8570 & 0.8484 & 1.4480 & 2.2303 & 3.6596 \\
$\left(\frac{3}{2}, 1\right)$ & 0.8455 & 1.2517 & 2.9144 & 2.0687 & 4.9666 \\
$\left(\frac{3}{2}, \frac{1}{2}\right)$ & 0.9435 & 1.3992 & 1.3987 & 3.8191 & 5.2095 \\
$(2,1)$ & 0.9264 & 1.8285 & 2.8350 & 3.6952 & 6.5197 \\
$\left(2, \frac{1}{2}\right)$ & 0.9691 & 1.9236 & 1.3789 & 5.3623 & 6.7367 \\
$\left(\frac{5}{2}, \frac{1}{2}\right)$ & 0.9804 & 2.4382 & 1.3683 & 6.8885 & 8.2540 \\
\hline \hline
\end{tabular}

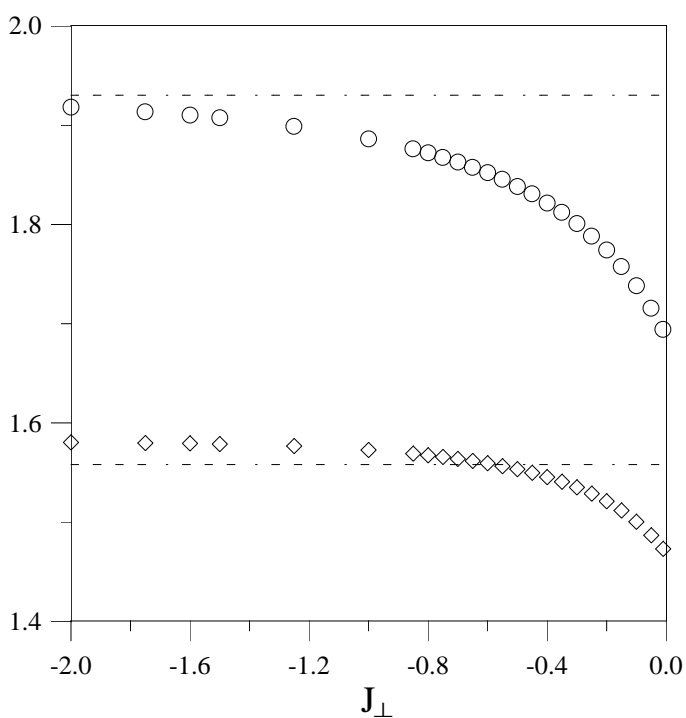

FIG. 7. Short-range intrachain correlations in the spin-singlet phase of the $\left(1, \frac{1}{2}\right)$ checkerboard ladder. The diamonds and circles represent, respectively, Lanczos numerical results $(N=10$ ladder $)$ for the spin-spin correlators $\left\langle\left[\mathbf{S}_{1}(n)+\mathbf{S}_{2}(n)\right] \cdot\left[\mathbf{S}_{1}(n+2)+\mathbf{S}_{2}(n+2)\right]\right\rangle$ and $\left\langle\left[\mathbf{S}_{1}(n)+\mathbf{S}_{2}(n)\right] \cdot\left[\mathbf{S}_{1}(n+4)+\mathbf{S}_{2}(n+4)\right]\right\rangle$ The dashed lines indicate $\mathrm{ED}$ results $(N=10)$ for the respective spin-spin correlators in the antiferromagnetic spin- $\frac{3}{2}$ Heisenberg chain.

In conclusion, the accomplished analysis clealy shows two basic implications of the underlying lattice structure for the ladder ferrimagnetic state: (i) the spin-wave excitations form folded acoustic and optical branches in the extended Brillouin zone and (ii) the ground state parameters (such as the on-site magnetizations $m_{A}$ and the spinstiffness constant $\rho_{s}$ ) show a crossover behavior in the weak-coupling region $0<J_{\perp}<1$. The presented results can be used in studies concerning the low-temperature thermodynamics of ferrimagnetic ladders. 


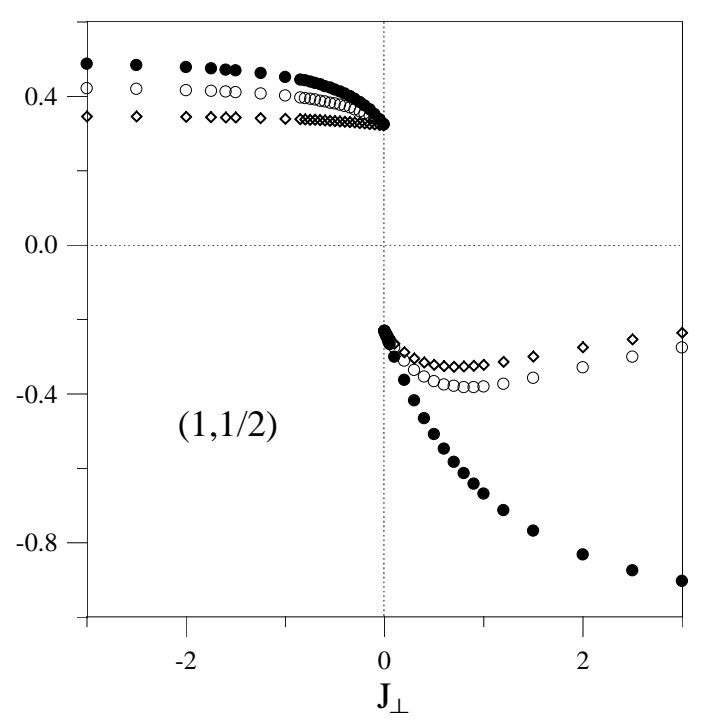

FIG. 8. Short-range interchain correlations in the $\left(1, \frac{1}{2}\right)$ system, as obtained from the numerical diagonalization of the $N=10$ ladder: $\left\langle\mathbf{S}_{1}(n) \cdot \mathbf{S}_{2}(n)\right\rangle$-filled circles, $\left\langle\mathbf{S}_{1}(n) \cdot \mathbf{S}_{2}(n+2)\right\rangle$-open circles, $\left\langle\mathbf{S}_{1}(n) \cdot \mathbf{S}_{2}(n+4)\right\rangle$-diamonds.

\section{ACKNOWLEDGMENTS}

We are grateful to Jörg Schulenburg for kindly providing us with his code for numerical diagonalization. This work was supported by the Deutsche Forschungsgemeinschaft (Grant No. 436BUL 113/106/1 and 106/2-1) and the Bulgarian Science Foundation (Grant No. F817/98).

* Permanent address: Institute of Solid State Physics, Bulgarian Academy of Sciences, Tzarigradsko chaussee-72, 1784 Sofia, Bulgaria.

${ }^{1}$ E. Dagotto and T.M. Rice, Science 271, 618 (1996), and references therein.

${ }^{2}$ S.R. White, R.M. Noack, and D.J. Scalapino, Phys. Rev. Lett. 73, 886 (1994).

${ }^{3}$ K. Totsuka, Phys. Rev. B 57, 3454 (1998).

${ }^{4}$ T. Giamarchi and A. M. Tsvelik, Phys. Rev. B 59, 11389 (1999).

${ }^{5}$ O. Kahn, Y. Pei and Y. Journaux, in Inorganic Materials (edited by D.W. Bruce and D. O'Hare, John Wiley \& Sons Ltd, New York, 1992).

${ }^{6}$ T. Fukui and N. Kawakami, J. Phys. Soc. Jpn. , 3454 (1998); Phys. Rev. B 57, 398 (1998).

${ }^{7}$ A. Koga, S. Kumada, N. Kawakami, and T. Fukui, J. Phys. Soc. Jpn. 67, 622 (1998).

${ }^{8}$ A. Langari, M. Abolfath, and M.A. Martin-Delgado, Phys. Rev. B 61, 343 (2000).

${ }^{9}$ A. Langari and M.A. Martin-Delgado, Phys. Rev. B 62, 11 725 (2000).
${ }^{10}$ E.H. Lieb and D. Mattis, J. Math. Phys. 3, 749 (1962); E.H. Lieb, Phys. Rev. Lett. 62, 1201 (1989); ibid. 62, 1927(E) (1989).

${ }^{11}$ N. B. Ivanov, Phys. Rev. B 57, R14 024 (1998).

12 S. Yamamoto, T. Fukui, and T. Sakai, Eur. Phys. J. B 15, 211 (2000).

13 N. B. Ivanov, Phys. Rev. B 62, 3271 (2000).

14 D. Sénéchal, Phys. Rev. B 52, 15319 (1995).

${ }^{15}$ A.B. Harris, D. Kumar, B.I. Halperin, and P.C. Hohenberg, Phys. Rev. B 3, 961 (1971).

${ }^{16}$ For details concerning the derivation of the bosonic quasiparticle Hamiltonian, see Ref. 13. Note that the magnetic unit cell connected to the classical ferrimagnetic state contains four spins, so that in the general case one would need four bosonic operators. However, due to the model symmetries, here it is possible to use only two bosonic operators, thus considerably reducing the following calculations.

17 T. Oguchi, Phys. Rev. 117, 117 (1960).

${ }^{18} V_{D M}$ and the related vertex functions may be obtained from the Dyson-Maleev interaction of the Heisenber ferrimagnetic chain (Ref. 13) by using the formal substitution $\gamma_{k} \mapsto \gamma_{k}+J_{\perp} / 2$.

19 The finite-size effect is small and can roughly be estimated from the density matrix renormalization group result at $J_{\perp}=0: m_{A}=0.79248$ [S.K. Pati, S. Ramasesha, and D. Sen, Phys. Rev. B 55, 8894 (1997)]. The ED result for $N=12$ is $m_{A}=0.79192$.

${ }^{20}$ In defining a spin-stiffness parameter for the ferrimagnetic state, we follow the classical work: B. I. Halperin and P. C. Hohenberg, Phys. Rev. 188, 898 (1969).

${ }^{21}$ F. C. Alcaraz and A. L. Malvezzi, J. Phys. A: Math. Gen. 30, 767 (1997).

22 N. Read and S. Sachdev, Phys. Rev. Lett. 75, 3509 (1995).

23 T. Barnes, E. Dagotto, J. Riera, and E.S. Swanson, Phys. Rev. B 47, 3196 (1993).

${ }^{24}$ S. Yamamoto, S. Brehmer, and H.-J. Mikeska, Phys. Rev. B 57, 13610 (1998).

${ }^{25}$ The indicated gap problem for the acoustic branch $E_{k}^{(\alpha)}$ in ferrimagnetic chains (Ref. 12) is an artifact of the nonsystematic treatment of quasiparticle interactions.

${ }^{26}$ Working at the level of free quasiparticles, one might draw just the opposite (incorrect) conclusion that the spin-wave series are more efficient for the ferromagnetic branch.

${ }^{27}$ From this perspective, the existence of a "ferrimagnetic state" in the regoin $-1<J_{\perp}<0$ (Ref. 8) seems puzzling.

${ }^{28}$ A.K. Kolezhuk and H.-J. Mikeska, Phys. Rev. B 53, R8848 (1996).

${ }^{29}$ M. Roji and S. Miyashita, J. Phys. Soc. Jpn. 65, 883 (1996).

\section{APPENDIX A: SECOND-ORDER CORRECTIONS FOR $M_{A}$}

The second-order correction for the on-site magnetization $m_{A}$ can be expressed in the form 
$m_{A}^{(2)}=-\frac{1}{2 N} \sum_{k}\left\langle\alpha_{k}^{\dagger} \alpha_{k}+\beta_{k}^{\dagger} \beta_{k}-\frac{\eta_{k}}{\varepsilon_{k}}\left(\alpha_{k}^{\dagger} \beta_{k}^{\dagger}+\alpha_{k} \beta_{k}\right)\right\rangle^{(2)}, \quad+\frac{2}{N} \sum_{q} \frac{V_{q}^{(-)} V_{q k ; k q}^{(4)}+V_{q}^{(+)} V_{k q ; q k}^{(4)}}{\left(\omega_{k}^{(\alpha)}+\omega_{k}^{(\beta)}\right)\left(\omega_{q}^{(\alpha)}+\omega_{q}^{(\beta)}\right)}$

where $\langle A\rangle^{(2)}$ denotes the second-order correction for the ground-state average of the operator $A$. The diagrams connected to $\left\langle\alpha_{k}^{\dagger} \alpha_{k}\right\rangle^{(2)},\left\langle\beta_{k}^{\dagger} \beta_{k}\right\rangle^{(2)},\left\langle\alpha_{k}^{\dagger} \beta_{k}^{\dagger}\right\rangle^{(2)}$, and $\left\langle\alpha_{k} \beta_{k}\right\rangle^{(2)}$ are presented in Fig. 9. The related explicit expressions read

$$
\begin{gathered}
\left\langle\alpha_{k}^{\dagger} \alpha_{k}\right\rangle^{(2)}=\frac{1}{(2 S)^{2}} \frac{V_{k}^{(+)} V_{k}^{(-)}}{\left(\omega_{k}^{(\alpha)}+\omega_{k}^{(\beta)}\right)^{2}} \\
+\frac{1}{(2 S)^{2}} \frac{2}{N^{2}} \sum_{2-4} \delta_{k 2}^{34} \frac{V_{43 ; 2 k}^{(8)} V_{k 2 ; 34}^{(7)}}{\left(\omega_{k}^{(\alpha)}+\omega_{2}^{(\alpha)}+\omega_{3}^{(\beta)}+\omega_{4}^{(\beta)}\right)^{2}} \\
\left\langle\beta_{k}^{\dagger} \beta_{k}\right\rangle^{(2)}=\frac{1}{(2 S)^{2}} \frac{V_{k}^{(+)} V_{k}^{(-)}}{\left(\omega_{k}^{(\alpha)}+\omega_{k}^{(\beta)}\right)^{2}} \\
\left\langle\frac{1}{(2 S)^{2}} \frac{2}{N^{2}} \sum_{2-4} \delta_{k 2}^{34} \frac{V_{43 ; 2 k}^{(7)} V_{k 2 ; 34}^{(8)}}{\left(\omega_{k}^{(\beta)}+\omega_{2}^{(\beta)}+\omega_{3}^{(\alpha)}+\omega_{4}^{(\alpha)}\right)^{2}},\right. \\
{\left[\frac{2}{N} \sum_{q} \frac{\mathrm{A}}{\left.\beta_{k}^{\dagger}+\alpha_{k} \beta_{k}\right\rangle^{(2)}=-\frac{1}{(2 S)^{2}} \times}\right.} \\
+\frac{2}{N} \sum_{q} \frac{\left.V_{q}^{(-)} V_{k q ; q k}^{(7)}+V_{q}^{(+)} V_{k q ; q k}^{(8)}+\omega_{k}^{(\beta)}\right)\left(\omega_{k}^{(\alpha)}+\omega_{q}^{(\alpha)}+\omega_{k}^{(\beta)}+\omega_{q}^{(\beta)}\right)}{\left(\omega_{q}^{(\alpha)}+\omega_{q}^{(\beta)}\right)\left(\omega_{k}^{(\alpha)}+\omega_{q}^{(\alpha)}+\omega_{k}^{(\beta)}+\omega_{q}^{(\beta)}\right)}
\end{gathered}
$$

$$
\begin{aligned}
& -\frac{2}{N^{2}} \sum_{2-4} \frac{\delta_{k 2}^{34}\left(V_{k 2 ; 34}^{(2)} V_{43 ; 2 k}^{(7)}+V_{k 2 ; 34}^{(8)} V_{43 ; 2 k}^{(3)}\right)}{\left(\omega_{k}^{(\alpha)}+\omega_{k}^{(\beta)}\right)\left(\omega_{k}^{(\beta)}+\omega_{2}^{(\beta)}+\omega_{3}^{(\alpha)}+\omega_{4}^{(\alpha)}\right)} \\
& \left.-\frac{2}{N^{2}} \sum_{2-4} \frac{\delta_{k 2}^{34}\left(V_{k 2 ; 34}^{(7)} V_{43 ; 2 k}^{(5)}+V_{k 2 ; 34}^{(6)} V_{43 ; 2 k}^{(8)}\right)}{\left(\omega_{k}^{(\alpha)}+\omega_{k}^{(\beta)}\right)\left(\omega_{k}^{(\alpha)}+\omega_{2}^{(\alpha)}+\omega_{3}^{(\beta)}+\omega_{4}^{(\beta)}\right)}\right] .
\end{aligned}
$$

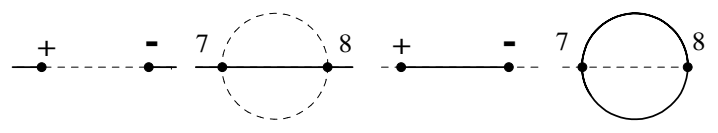

$\left(a_{1}\right) \quad\left(a_{2}\right) \quad\left(b_{1}\right) \quad\left(b_{2}\right)$

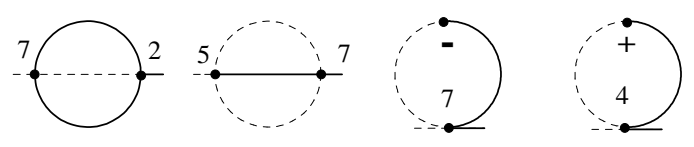

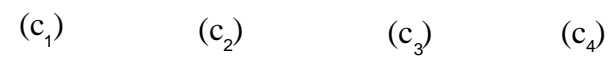

FIG. 9. Second-order diagrams contributing to $m_{A}^{(2)}$ : (a), (b), and (c) diagrams give contributions, respectively, to $\left\langle\alpha_{k}^{\dagger} \alpha_{k}\right\rangle^{(2)},\left\langle\beta_{k}^{\dagger} \beta_{k}\right\rangle^{(2)}$, and $\left\langle\alpha_{k} \beta_{k}\right\rangle^{(2)}$. The diagrams for $\left\langle\alpha_{k}^{\dagger} \beta_{k}^{\dagger}\right\rangle^{(2)}$ can be obtained from the (c) diagrams by using the following vertex substitutions: $c_{1}:(7,2) \rightarrow(8,3), c_{2}$ : $(5,7) \rightarrow(6,8), c_{3}:(-, 7) \rightarrow(+, 8)$, and $c_{4}:(+, 4) \rightarrow(-, 4)$. The $\alpha(\beta)$ magnon propagators are represented by solid (dashed) lines, whereas the vertex functions are denoted by their superscript indices. 